\title{
Comparison of the 3 different levels of PEEP on haemodynamics, respiration and arterial oxygenation; which is the best PEEP for sleeve gastrectomy under general anaesthesia?
}

\author{
Huseyinoglu K., Goksu S., Bakan N. \\ Health Science University of Umraniye Training and Education Hospital \\ Istanbul, Turkey
}

Background: In this prospective randomised study, our goal is to compare the effects of 3 different PEEP levels $(0-5-10 \mathrm{~cm}$ $\mathrm{H}_{2} \mathrm{O}$ ) on hemodynamic parameters in order to evaluate their effects on respiration and arterial oxygenation in patients undergoing laparoscopic bariatric surgery of obese patient (sleeve gastrectomy) under general anesthesia.

Methods: After approval of local ethics committee and patient consent, 60 adult patients with a body mass index (BMI) of $>40$ with American Society of Anesthesiologists group I-II were randomised into three groups according to aplied PEEP level: Group I; zero PEEP.

Group II; $5 \mathrm{cmH}_{2} \mathrm{O}$ PEEP and

Group III; $10 \mathrm{cmH}_{2} \mathrm{O}$ PEEP.

All other variables (e.g., anesthetic and surgical techniques and ventilatory settings) were the same for all patients. Duration time, heart rate, invasive arterial blood pressures, arterial blood gases (ABG), Ppeak, Pplateau, $\mathrm{SpO}_{2}$ and

$\mathrm{ETCO}_{2}$ were measured intraoperatively and postoperatively in the postanesthesia care unit (PACU). IBM SPSS statistics 22 (IBM SPSS Turkey), Shapiro Wilks test, One-way anova test, Tukey HSD, Tamhane's T2 test, Kruskal Wallis, Mann Whiyney $U$, paired sample t and chi-square tests were used for statistical anlyses. A value of $p<0.05$ was considered to be significant.

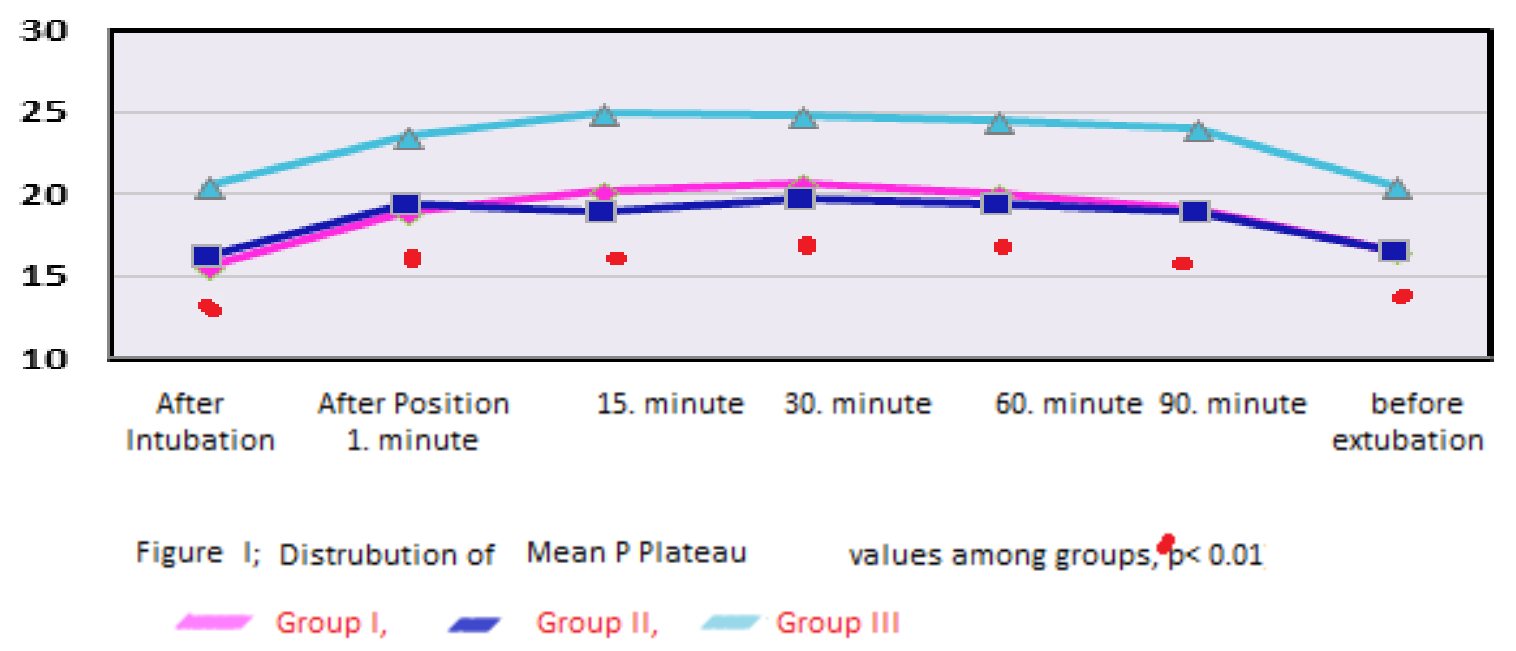

Results;

The study conducted 60 patient

( $11.7 \%$ males and $88.3 \%$ females and mean age of $37.8 \pm 10.81$, BMI ranged between 41,58 - $51.7 \quad \mathrm{~kg} / \mathrm{m}^{2} \quad$ ). There was no difference between groups with respect of the demographic data,

operation time,

$\mathrm{ABG}$,

Ppeak and

Hemodynamic measurements.

At all groups significant increases were detected in PPlateau at all times compared to initial value and among groups Group III values were always higher than other groups $(p<0.01)$ (Figure I). The statical compliances of group II and group III were significantly higher than group I at all times $(p<0.01)$ ( Figure II). After intubation, after position and before extubation values, Group I's $\mathrm{pO}_{2}$ values was significantly lower than other groups, after extubation returned to nornal $(p<0.01)$ (Table 1$)$.

Conclusion; We suggest that $5 \mathrm{~cm} \mathrm{H}_{2} \mathrm{O}$ PEEP is optimal PEEP in sleeve gastrectomy cases amoung this 3 different PEEP level in this study, because it causes a significant increas in compliance without affecting $P$ peak and slightly increase in $P$ Plateau.

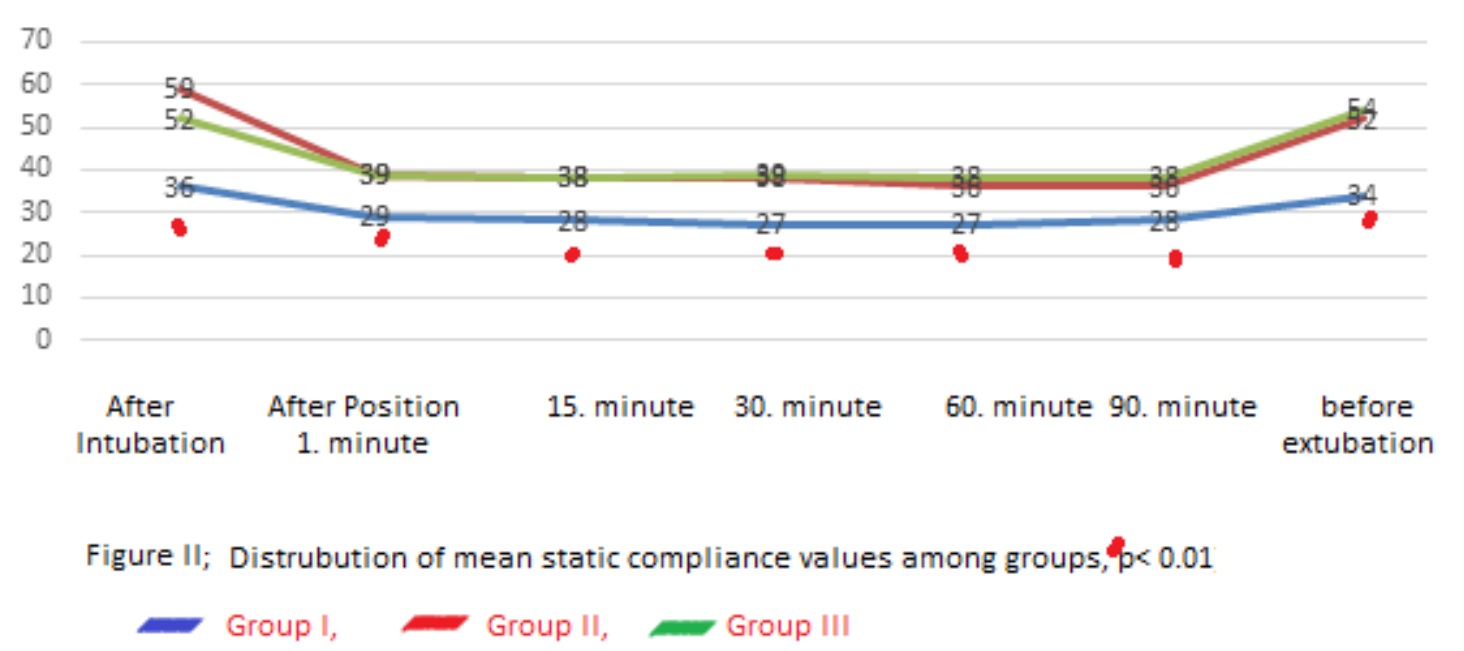

Figure 1; Arterial blood sample results. One-Way Anova ${ }^{++} p<0.01$

\begin{tabular}{|c|c|c|c|c|c|}
\hline & Grup I & \multirow{2}{*}{\begin{tabular}{|l} 
Grup II \\
Mean \pm Sd
\end{tabular}} & \multirow{2}{*}{$\begin{array}{l}\text { Grup III } \\
\text { Mean } \pm \text { Sd }\end{array}$} & \multirow[t]{2}{*}{${ }^{a} \mathbf{p}$} \\
\hline & & Mean $\pm S d$ & & & \\
\hline \multirow[t]{8}{*}{$\mathrm{pH}$} & After Intubation & $7.43 \pm 0.04$ & $7.41 \pm 0.06$ & $7.43 \pm 0.05$ & 0.358 \\
\hline & After Position & $7.40 \pm 0.04$ & $7.40 \pm 0.05$ & $7.40 \pm 0.05$ & 0.980 \\
\hline & Before Ekstubation & $7.37 \pm 0.03$ & $7.36 \pm 0.05$ & $7.37 \pm 0.05$ & 0.676 \\
\hline & $30 \mathrm{~min}$. after procedure & $7.36 \pm 0.04$ & $7.34 \pm 0.05$ & $7.35 \pm 0.05$ & 0.348 \\
\hline & After Intubation & $181.49 \pm 39.25$ & $246.01 \pm 52.44$ & $254.42 \pm 43.07$ & $0,001^{+\dagger}$ \\
\hline & After Position & $191.70 \pm 29.47$ & $235.36 \pm 66.68$ & $234.78 \pm 35.22$ & $0.006^{+\dagger}$ \\
\hline & Before Ekstubation & $216.30 \pm 55.98$ & $262.09+74.31$ & $246.07 \pm 49.92$ & $0.042^{+}$ \\
\hline & $30 \mathrm{~min}$. after procedure & $228.99 \pm 126.58$ & $271.61 \pm 54.32$ & $260.43 \pm 70.50$ & 0.415 \\
\hline \multirow{5}{*}{$\mathrm{pCO}_{2}$} & After Intubation & $35.34 \pm 3.65$ & $33.73 \pm 5.09$ & $33.30 \pm 3.60$ & 0.281 \\
\hline & After Position & $36.17 \pm 4.47$ & $33.67 \pm 50$ & $35.92 \pm 4.08$ & 0.129 \\
\hline & Before Ekstubation & $37.96 \pm 3.45$ & $37.38 \pm 4.27$ & $37.42 \pm 4.57$ & 0.885 \\
\hline & $30 \mathrm{~min}$. after procedure & & & & 0.803 \\
\hline & & $38.31 \pm 3.72$ & $37.81 \pm 4.41$ & $37.42 \pm 4.50$ & \\
\hline \multirow[t]{4}{*}{$\mathrm{SPO}_{2}$} & After Intubation & $98.99 \pm 0.95$ & $98.97 \pm 1.93$ & $99.05 \pm 0.44$ & 0.425 \\
\hline & After Position & $98.85 \pm 0.98$ & $99.06 \pm 0.87$ & $98.99 \pm 0.90$ & 0.756 \\
\hline & Before Ekstubation & $99.02 \pm 0.70$ & $99.13 \pm 0.59$ & $98.76 \pm 1.35$ & 0.435 \\
\hline & $30 \mathrm{~min}$. after procedure & $98.09 \pm 2.37$ & $98.50 \pm 1.56$ & $99.36 \pm 0.83$ & 0.155 \\
\hline
\end{tabular}

